\title{
Rx: Observe, Do not Treat When is it Appropriate not to Treat?
}

\section{David V Gauvin ${ }^{1^{*}}$ and Scott E Boley ${ }^{2}$}

${ }^{1}$ Neurobehavioral Sciences, MPI Research, Mattawan, MI, USA

${ }^{2}$ Drug Safety Assessment, MPI Research, Mattawan, MI, USA

*Corresponding author: David V Gauvin, Neurobehavioral Sciences, MPI Research, 54943 North Main St., Mattawan, MI, USA, Tel: +1 269-668-3336; E-mail: david.gauvin@mpiresearch.com

Received Date: Nov 02, 2017, Accepted Date: Nov 16, 2017, Publish Date: Nov 28, 2017

Copyright: ( 2017 Gauvin DV, et al. This is an open-access article distributed under the terms of the Creative Commons Attribution License, which permits unrestricted use, distribution, and reproduction in any medium, provided the original author and source are credited.

\begin{abstract}
Development of a protocol for a nonclinical safety study to evaluate the safety profile of an investigational therapeutic, whether it is a toxicology or safety pharmacology study, must not be conducted in haste. The evaluation of this novel therapeutics relies on the integrated strategy developed by the scientific team, which may include the pharmaceutical company and the services of a contract research organization. All nonclinical study protocols involving the use of live animals require the inclusion of the Institutional Animal Care and Use Committee (IACUC) and staff veterinarians during the review of the study protocol. The team, as a whole, must endure a frank, honest, and open discussion regarding study design and animal welfare issues. All available information regarding the test material should be shared with all parties in a manner and time that allows for constructive protocol and treatment strategy development. The intent of nonclinical safety assessments is driven by both administrative guidelines from drug regulatory agencies and statutory (legal) controls of federal laws. In working with test articles, particularly for small molecules, the probability of unexpected findings is relatively high. The research team must maintain the highest standards of animal care throughout this process which is complicated by the fact that when animal health and welfare issues occur they must be remedied fast, efficiently, and transparently. We review the pitfalls of safety assessment strategies and offer some industry standard resolutions that may help to make the road to market a little easier. The paper is written based on small molecule development, but many of the points would also apply to biopharmaceuticals as well.
\end{abstract}

Keywords: Safety assessments; Toxicology studies; IND-enabling studies; Animal welfare; Nonclinical studies

\section{Introduction}

Nonclinical safety testing serves a fundamental role in characterizing the potential clinical risks associated with test articles submitted to the FDA for review prior to human use. There have been serious and sometimes rare and unexpected adverse events in clinical trials or post-approval, suggesting that critical gaps may exist in our understanding of the relationship between human response and nonclinical toxicology findings. For example, non-clinical safety assessments are conducted in normal healthy test systems and are designed to be "exposure-based"; it does not attempt to evaluate potential risk of rare or idiosyncratic pharmacological responses that may arise from interactions with the presence or progression of disease, genetic variability, or other pharmacological exposures of human patients. Additionally, the true predictive accuracy of many toxicology models and safety pharmacology assays can be uncertain relative to actual human risk.

Two of the most difficult but critical problems in risk assessment are defining the equivalent human dose and determining the doseresponse curve below the dose region that induces adverse events [1]. If the only information available to drug approval regulators is the applied or external dose, the estimated human equivalency dose will be based on several assumptions, such as equivalent absorption in laboratory animals and humans. Uncertainties may exist in the predicted low-dose effects in humans if 1) all of the doses tested in the nonclinical toxicity study protocols caused adverse effects that were above the metabolic saturation exposure, 2) if the test article is absorbed differently in animals than in humans, or 3) if the test article is metabolized differently (qualitatively or quantitatively) between species in a significant manner.

An objective of these early animal studies is to improve our understanding of the relationship between drug exposure and the effects it has on the test system. By increasing our understanding of the relationships between systemic exposure and adverse effects, we increase our confidence in understanding the possible human risk. Thus, knowledge of actual test article plasma concentrations and factors that influence absorption, distribution, metabolism, and elimination in experimental animals and how that relates to humans provides a greater scientific rationale for estimating risk than do estimates that are based solely on adverse effects or death. Exposureand time-dependent changes in exposure for a parent compound and its metabolite(s) can provide an even stronger scientific foundation for estimating the human dose equivalent in various risk assessment models, especially if the adverse effects of exposure are due to particular metabolites (e.g. ethyl alcohol and acetaminophen).

Under the U.S. Food and Drug Administration's Good Laboratory Practice Guidelines (GLPs, \$58.3) a nonclinical laboratory study means in vivo or in vitro experiments in which test articles are studied prospectively in test systems (animals/cells) under laboratory conditions to determine their safety. Under the GLPs (\$58.31) the test facility management must identify and select a central point of control for each nonclinical study conducted within the research institution. The Study Director (\$58.33) is a scientist, or other professional, of appropriate education, training, and experience, or combination thereof, that has overall responsibility for the technical conduct of the 
study, as well as for the interpretation, analysis, documentation and reporting of results, thereby representing a single point of study control. The guidelines do not restrict or require any specific attributes of education, background or experience for the individual identified as the Study Director (SD), but it is the responsibility of test facility management to assure the SD is capable of performing the job.

\section{Where is the Cyclone?}

An area of significant consideration for nonclinical safety assessment studies is the health and welfare of the test systems since valid and reliable data is contingent upon a healthy experimental subject. Procedures for animal husbandry, dose administrations, health status observations, tissue harvesting, and termination of animals must be approved by the IACUC in accordance with the Animal Welfare Act (7 U.S.C. 2131, et seq.). In cases where funds are received from the Public Health Service (PHS), procedures must also comply with the PHS Policy on Humane Care and Use of Laboratory Animals, according to section 495 of the PHS Act (42 U.S.C. 289(d)). While not specifically required by statutes or administrative regulations, it is highly recommended that the safety assessment studies be conducted by research facilities that are accredited by the Association of Assessment and Accreditation for Laboratory Animal Care (AAALAC).

By their nature and purpose nonclinical safety assessment studies must examine a full range of pharmacological activity up to and including toxicity of test articles that have no prior data or information as to any expected findings in a test system. Doses are selected in order to explore the toxicity of the test article and to help understand the mechanisms of toxicity up to and including the cause of death. Under the GLPs (\$58.90) at the initiation of a nonclinical laboratory study, animals must be free of any disease or condition that might interfere with the purpose or conduct of the study. If, during the course of the study, the animals exhibit a declining condition, they are typically placed under veterinary consultation. Under the GLPs, the animals may be treated while on study, provided that such treatment does not interfere with the study. The diagnosis, authorizations of treatment, description of treatment, and each date of treatment shall be documented and retained. This sets the stage for a "perfect storm" during the conduct of studies with animal subjects.

Misinformation about drug treatments can mislead the industry into underestimating the risks, or overestimating the benefits, of various veterinary interventions during the conduct of nonclinical toxicology studies. Even if information is correct for one study and one specific test article, the generalizability of such information to all other test articles with new mechanisms of action may not be appropriate. Traces of this type of misinformation (called "belief echoes") can persist and influence subsequent judgments and decisions during the conduct of other studies within a given facility. With the growing numbers of open forum chat rooms, blogs, and rapid communication journals these belief echo's may also influence other research facilities to adopt similar treatment strategies and consider them to be "standard", benign, or safe.

\section{Some classic examples of such interactions are:}

1. Metabolic syndrome is associated with several disorders including hypertension, diabetes, hyperlipidemia as well as cardiovascular diseases and stroke. In investigating a new veterinary treatment it should be understood that plant-derived polyphenols, found in numerous plant species, play an important role as potential treatments for components of metabolic syndrome. Studies have provided evidence for protective effects of various polyphenol-rich foods against metabolic syndrome. Standard food enrichments such as fruits, vegetables, cereals, nuts, and berries are rich in polyphenolic compounds [2]. Grapes ( Vitis vinifera), especially grape seeds, stand out as rich sources of antioxidants and have been reported to inhibit the risk factors involved in metabolic syndrome such as hyperlipidemia, hyperglycemia, and hypertension in both humans and animals. It should be noted that under the GLPs $(\$ 58.90 \mathrm{~g})$ the feed and water used for the animals must be analyzed periodically to ensure that contaminants known to be capable of interfering with the study and reasonably expected to be present in such feed or water are not present at levels above those specified in the protocol. Food that is not certified to be free of contaminants cannot be used on a GLP compliant study without acknowledging the exception in the final report, but basic nutrient constituents of food enrichments are not considered contaminants. In this respect is it a reasonable expectation that SDs have knowledge of potential nutrient-drug interactions on studies and the possible impact on data variability and interpretation as a result of such use of food enrichments on studies?

2. One standard treatment usually considered benign and often used in nonclinical testing facilities when clinical signs of anaphylaxis are noted, is the administration of the antihistamine, diphenhydramine (Benadryl ${ }^{\circ}$ ). Histamine has a very potent contracting action on the sphincter and bile duct and this is antagonized by diphenhydramine. Pharmacologically, opiates also contract the bile duct and sphincter of Oddi. Opiate-induced contraction of the sphincter of Oddi and bile duct is mediated by a two-step reaction involving interaction with a specific opiate receptor leading to the release of histamine which combines with an $\mathrm{H} 1$ receptor to produce the effect [3]. Diphen-hydramine has also been shown to interact directly with the cytochrome P450 enzyme CYP2D6 [4]. Clinically relevant drug-drug interactions may occur between diphenhydramine and many CYP2D6 substrates, particularly those with a narrow therapeutic index. Depending on the test article metabolites can be formed in the liver by CYP2D6 enzymes and excreted into the intestinal tract through the sphincter of Oddi and bile ducts. These metabolites can be reabsorbed into the blood and ultimately excreted in the urine, or simply be excreted in the feces. The effectiveness of the liver as an excretory organ for glucuronide conjugates is limited by their enzymatic hydrolysis after the bile is mixed with the contents of the small intestines following a dose of diphenhydramine. Based on this information this commonly used veterinary intervention is not completely benign.

3. Injuries not related to the test article are common in laboratory bred animals housed in standard caging. One standard treatment for inflammation and analgesia commonly used for such injuries is meloxicam (Metacam ${ }^{\mathrm{TM}}$ ). The FDA has identified over 1300 approved drugs and their generic formulations that interact with a therapeutic dose of meloxicam. The sheer number of drug interactions for this standard treatment verifies the the possibility of inducing systemic variability within any group that receives such treatment when deciding to allow for administration of meloxicam on a study.

Investigations of a pharmaceutical's Absorption, Distribution, Metabolism, Elimination and Toxicology (ADMET) play a central role 
in the nonclinical and clinical safety assessment of human medicines. Active Pharmaceutical Ingredients (APIs) used in nonclinical safety assessment studies must be evaluated in at least two species which may, in fact, have differing enzymatic degradation pathways for the same API. Drug metabolism via the cytochrome P450 system is an important determinant in the possibility of drug-drug interactions. CYP450 enzymes represents a family of isoenzymes that are involved in the metabolism of many test articles as well as the activation of prodrugs. The enzymes are heme-containing membrane proteins, which are located in the smooth endoplasmic reticulum of several tissues. Although a majority of the isoenzymes are located in the liver, extrahepatic metabolism also occurs in the kidneys, skin, gastrointestinal tract, and lungs.

There are more than fifty CYP450 enzymes, but for humans the CYP1A2, CYP2C9, CYP2C19, CYP2D6, CYP3A4, and CYP3A5 enzymes metabolize $90 \%$ of drugs. In laboratory animals the CYP enzymes demonstrate species-specific differences from those in humans but their role in the context of a nonclinical study is the samemetabolizing test articles. These enzymes are predominantly expressed in the liver, but they also occur in the small intestine (increasing/ reducing drug bioavailability), lungs, placenta, and kidneys. Many drug interactions are the result of an alteration of CYP450 metabolism.

Adding a veterinary therapeutic agent during the conduct of a nonclinical study may act as either inhibitors or inducers of the CYP450 enzyme(s) responsible for test article metabolism. Enzyme induction is the process by which exposure to certain substrates (e.g., drugs) results in increased CYP450 activity and subsequent accelerated biotransformation with a corresponding reduction in un-metabolized test article, which can ultimately alter the effect of the test article on the animal. Most drugs can exhibit decreased efficacy due to rapid metabolism, but drugs with active metabolites can display increased drug effect and/or toxicity due to enzyme induction. Enzyme inhibition occurs when two drugs sharing metabolism through the same isoenzyme compete for the same enzyme receptor site. The more potent inhibitor will result in decreased metabolism of the competing drug. For most drugs, this can lead to increased serum levels of the unmetabolized entity, and possibly increased toxicity. For drugs whose pharmacological activity requires biotransformation from a pro-drug form, inhibition of CYP450 enzyme(s) can lead to decreased efficacy. Inhibition or induction of CYP450 enzymes is most concerning for test articles that have a steep dose-response between no effects and significant toxicity since small changes in serum levels of the test article could have substantial effects on the animal.

A test article can be both metabolized by, and inhibit, the same enzyme (e.g., erythromycin), or it can be metabolized by one enzyme and inhibit another enzyme (e.g., terbinafine [Lamisil]). Standard veterinary intervention drug doses may cause unintended effects related to altered serum levels of the test article if an animal is a poor metabolizer or the veterinary treatment impacts the CYP450 enzyme responsible for metabolizing the test article. Adverse effects are more likely to occur if a test article of interest has a narrow safety range or is metabolized by only one enzyme and that enzyme is impacted by the veterinary treatment.

The real problem with veterinary drugs that share enzymatic pathways with the test article is that this is usually not discovered until an issue develops. When the drug-drug interaction results in exposures reaching a toxic state, the toxicity can manifest itself with serious medical consequences. A classic example occurred with the proarrhythmic effects (QT prolongation) from high serum levels of non- sedating antihistamines (terfenadine and astemizole) with an antibiotic (erythromycin) which severely limited their usefulness and led to the development of newer agents to take their place.

On the other side of the coin, from the veterinarian's perspective, meeting animal welfare compliance expectations for providing veterinary care or euthanizing animals in distress has presented challenges in balancing this need against questions of whether the proposed veterinary care would interfere with study objectives. From our experience when animals are exhibiting significant signs of toxicity most veterinarians would opt for euthanasia over treatment, if allowed. However, if early euthanasia is not consistent with study goals, an alternate approach is needed. The most consistent approach to safety assessment from the veterinary perspective is to use additional means of identifying when severe toxicity is developing and establishing criteria for euthanasia before the animal reaches a moribund state (i.e., a humane endpoint). The general concept has been that this is good science, as identifying clinical pathology changes or other biomarkers for severe toxicity aides in developing the toxicity profile for a test article. Aside from animal welfare, it can also validate biomarkers for clinical trials, and allow for necropsy and tissue evaluation prior to an animal being found dead.

The acute/dose range finding toxicology assays have undergone significant study design changes over the last 5 to 10 years. To start the whole process the current approach to these studies uses a flexible protocol: either 'up and down' for small animals or 'ascending dose' for large animals. Although the goal of these studies have always been to define a Maximum Tolerated Dose (MTD), the studies have evolved to use fewer animals and instead of focusing on "full dose-response evaluations" the current strategy is to provide a limited set of data that establishes a rough range of effects. The target is no longer mortality or moribundity; the goal is to maintain survival while exploring the limits of toxicity. Another goal of these and subsequent toxicology studies is to define an adverse dose level, however the term "adverse effect" has been the point of continual debate within the industry. An adverse effect has been defined as a diminished ability of the animal to perform a critical function of daily living, such as failure to groom, failure to eat, etc. [5]. Others refer to an adverse health effect as one where there are unmistakable health effects that cause functional impairment in organ systems (liver, kidney, etc.) that will further increase susceptibility to disease or morbidity. The diminished ability is always deleterious, regardless of the subsequent survivability. The utility of this definition is that it focusses on a specific ability, such as strength, coordinated movements, hearing, attention, etc which can be inferred from observations or actual measures of behavior. If the basis for describing an animal as "sick" is a change in behavior, it then becomes circular to explain the change in behavior as the basis of a concept whose only evidence for existence is that change in behaviour [6]. In this example, veterinary intervention to minimize distress and maintain survivability typically has little impact on determining an MTD, however it could impact determining an adverse effect level. Mechanistic understanding of the expression of clinical and behavioral signs of toxicity are the basis of identifying key molecular "triggers" that can alter biological processes and ultimately lead to toxicity. This understanding should not be critically altered by allowing veterinary interventions that will reduce anxiety, stress, and distress of animals on study.

It is extremely troublesome within the industry that Congress injected confusion and debate into the regulatory approval process by inserting the term "psychological well-being" into the law books when 
it wrote and passed the AWA without a statutory (legal) definition. A myriad of views and definitions have been used in the discussion of stress and distress. While there is little denial on either side of the veterinary intervention argument that stress is a real or perceived threat to an animal's physiological homeostasis, defining "animal wellbeing" without relying on some form of anthropomorphic measures is a challenge to both regulators and researchers. Over the last 20 years of conducting research under the AWA the scientific, regulatory, and animal care communities still disagree with respect to a universally accepted operational or lexical definition of distress. The National Research Council (NRC) has yet to support objective criteria or principles with which to qualify distress. The objective scientific assessment of subjective emotional states is yet to be proven and while there is often a measure of agreement on the interpretation of physiologic and/or behavioral variables as indicators of stress, distress, or welfare status, there has not always a direct link. The NRC's position is that even if a universally accepted definition existed, it could not be applied across all species and all conditions, because of the differential impact of the strain, age, gender, genetic background and environment [7]. When drug approval agencies cannot yet agree on these statutory control issues, a "double bind" dilemma is imposed onto the pharmaceutical registrants under the administrative review process of these agencies. It is up to federal regulatory agency administrators to recognize this dilemma and take the appropriate steps to address this situation. While FDA, DEA, EPA, and USDA guidelines are not legally binding on the agencies, or their registrants, all of us are legally bound under the law to follow the AWA.

Early safety assessments are typically initiated by conducting doserange finding studies mentioned previously. A typical single dose range-finding study (DRF) might include one animal/sex for nonrodents, or three animals/sex for rodents using at least three dose levels up to a "limit dose", typically 1000 or $2000 \mathrm{mg} / \mathrm{kg}$. The objective is to establish the MTD and support dose selection for subsequent studies. In these studies the MTD is typically based on clinical signs of toxicity and mortality; gross necropsy exams are not usually performed. The MTD is considered the dose that above which the animals would not tolerate the test article-related effects (mortality, convulsions etc...). Veterinary treatments to minimize the magnitude or duration clinical signs should not impact the determination of the MTD, but it can allow for the animal to survive the testing with reduced stress. Once effects supporting an MTD have been documented on a study the question then becomes what is the rationale for "pulling out all the stops" to retain moribund animals until the end of the study duration? We acknowledge that current regulatory guidelines for carcinogenicity studies do give high importance to the total number of animals retained to the end of the study duration, but that is based on the objectives of those study designs and this is not a generally accepted requirement for all other nonclinical safety assessment study designs.

"Distress" may not always manifest itself with recognizable "maladaptive behaviors such as abnormal feeding or aggression" [8] but instead begin with subclinical pathological changes (e.g. hypertension, immunosuppression, stereotypies, etc.) that can lead to overt toxicity. As the single point of control on a study it is the SD who must make the call to "treat or not to treat". Under the GLPs, the SD need not be trained in pharmacokinetics and may not know how the test article is metabolized, especially in the early stages of product development. However the same can be said about board-certified veterinarians at the same institutions. The veterinarians and clinical medicine support team may be focusing on animal welfare, health, or distress without the full knowledge or awareness of how their prescribed veterinary interventions may influence study outcomes. However, in today's research environment the principles of animal welfare seem to supersede the regulatory and statutory requirements of drug development. The clear definitions for stress, distress, and wellbeing must be well-defined within: 1) Standard Operating Procedures approved by Test Facility Management, 2) by legal or literature citation within contractual agreements between Sponsors and CROs, or 3) within the Study Protocol during study development.

There can be legitimate reasons to withhold aggressive medical interventions on study designs, but not all treatments are as "confounding" as others. When stress, distress, or well-being are in question, the research team needs to create a hierarchical treatment strategy plan and have it in place prior to the start of dosing as much as possible-this is in full compliance with administrative and statutory control directive (see below).

\section{Toto, we are not in Kansas anymore}

Just 15 or 20 years ago nonclinical research was conducted almost "carte blanche" with minimal oversight by the IACUC once the study protocol was approved. Not to be inflammatory, but to many researchers animal subjects were simply "petri dishes with legs". At the time of study initiation, there seemed to be little, or a minimal consideration for animal welfare during the study. Since the adoption of the Animal Welfare Act, the tide has shifted and animal welfare now predominates throughout the global regulatory agencies governing and monitoring the industry's study conduct. Governmental regulatory agencies and research facilities have acknowledged that valid and reliable data are contingent upon the health and well-being of experimental subjects. The quality of animal welfare within the research laboratory is contingent upon the quality of work and monitoring by those who conduct the research.

There continues to be friction with regard to the topic of veterinary interventions during the conduct of a nonclinical safety assessment study. "Old school" researchers see the value in "drug free" assessment strategies; to the seasoned toxicologist the core foundation of study design is to reduce variability whenever possible. To the seasoned nonclinical researcher history has revealed that all drugs have multiple effects and even the most benign veterinary interventions, proposed by a well-intentioned veterinarian, are often seen as introducing a variable and that poses a potential impact on the study data. Intervention of any type may have a potential to alter the pharmacokinetic or dynamic response to test article administration and have been bitterly opposed by many researchers. The view from the veterinary side of the issue has often been disregarded. An institutional conflict was established between the FDA's regulatory or administrative authority of the GLPs that gave the "veto power" of treatment authorization to the single point of study conduct-the SD and the federal statutory (Congressional) control of animal welfare established by the adoption of the AWA. Under the new paradigm, the Institutional Animal Care and Use Committee (IACUC) is the institutional authority for representing both the SD and animal welfare perspectives [9-12].

The staff veterinarian may have been demonized during the paradigm shift that was instituted by the statutory requirements of the AWA. However, over the last 5 to 10 years a careful review and revision of animal welfare policies and attitudes with regard to nonclinical safety studies seems to have occurred, resulting in a global paradigm shift. The current zeitgeist has opened the door to negotiations between veterinarians and nonclinical researchers. The civil disputes 
between Sponsors and CROs and between researchers and veterinarians have diminished. The theoretical threat to valid and reliable nonclinical data generation by veterinary interventions is now recognized and discussed prior to implementation. Both sides have managed to extend a set of common principles for study conduct that are functional, data-based, and scientifically sound.

\section{Ten Yellow Bricks on the Road to $\mathrm{Oz}$}

The integrated roles of SD, Sponsor Study Monitor, IACUC, and staff veterinarian are now critical for the development and execution of nonclinical safety assessments in the CRO. The "hands off" policies of old are now "all hands on board" and the development of an institutionalized open team policy of study conduct is not a pipedream. During study protocol development many of the common interests of all parties can be easily addressed so that when, or if, a veterinary intervention is proposed, or required, a minimal time to targeted treatment initiation can be documented. Here are some starting points.

1. All parties involved in study design should acknowledge that working with laboratory bred animals is a privilege and a requirement by international and national policies and statutes. We do what we do, because it is the law.

2. Animal health and well-being of the experimental subjects is non-negotiable, it too is the law (AWA) and absolutely critical to the success of a nonclinical study.

3. Witholding treatment on study should not be a standard for any study conducted with live animals, the time for that mantra has passed. Treatments are not necessarily restricted to pharmacological intervention and can include observations, wound care, etc. If an animal is experiencing pain or distress that can't be treated the only alternative is euthanasia. If the SD desires to withhold veterinary care or euthanasia, they need approval by the IACUC first and they must have a sound scientific rationale.

4. However, it is acknowledged that all veterinary interventions have a potential to modify ongoing behaviors, physiological processes, and pharmacological responses to test article administrations. When we need to act we must act judiciously and with forethought. 4a) A proposed treatment plan for veterinary intervention is essential and part of protocol development. Many options are available that allow for full compliance to both administrative guidelines and statutory codes. For example, if stereotypical allogrooming in a rat is expected following dose administrations, the first line of treatment may simply be to add environmental or food enrichments in the cage as distractors from such behaviors. If the behavior escalates, the repetitive grooming may induce selfinjury or barbering. The treatment may then become more aggressive and shift to twice daily observations with topical cleaning of all visible wounds with an approved antiseptic/ cleaning agent, such as Nolvasan or chlorhexidine solutions. Depending on the duration of the study, the next step may include an "Elizabethan collar" or "skirt" to physically prevent access and further tissue injury in the hopes that behavioral tolerance may be learned to reduce the likelihood of further progression. All of these options should be addressed before protocol authorization or dose administration.

5. All parties involved in study development, especially at CROs, are governed by conflict-of-interest and confidentiality contracts, we are all on the same team. A scientifically sound study design requires valid and reliable data regarding the test article, which means all information on an test article should be shared between the parties. 5a) The Sponsor must provide sufficient information, data, knowledge, or proposed "theories" of mechanisms of action during the process of protocol development so that all interested parties are working from the same level of information. 5b) Sufficient time should be given to allow for SD, staff veterinarians, and Sponsor Monitors to work through any proposed treatment strategies. At the nonclinical level of research "double blinded" dose administration should be limited to the animal technician level. SD and veterinary interventionists at all levels should have full disclosure.

6. A veterinary intervention strategy should be in place prior to the start of dosing; this can be part of the protocol or as an IACUC/ institutional policy. Everyone in the research team should have equivalent knowledge about the test article regarding expected pharmacology. This refers to questions regarding peripheral versus central nervous system effects; proposed absorption, distribution, excretion and metabolic profiles consistent with the pharmacological class or therapeutic targets.

7. IACUC approval should be contingent upon sounds scientific justification of test systems (species selection), dose levels, dose durations, as well as clinical signs of toxicity or adversity that would initiate an intervention plan. 7a) The pace, cadence, and rhythm of daily research activities plays a factor in veterinary interventions; hesitation in treatment approvals must be minimized. The delay to contact a Sponsor Monitor to discuss options at the time of an unexpected incident or injury must be minimized. As the single point of control, the SD must know their options at study initiation. Under the animal welfare regulations, only the IACUC may approve withholding of treatment for pain or distress, the veterinarian does not have this authority. The SD may request approval for restrictions in veterinary care if there is sound scientific justification for withholding treatment or even early euthanasia. However, such justifications are closely scrutinized and humane endpoints should be sought whenever possible.

8. The group size $(\mathrm{N})$ of any study should have enough power to allow for subject loss without compromising statistical power. Treatment of one or two animals in any group should not compromise a study-expect interventions. Do not accept the possibility of an injury free study in laboratory bred animals since living organisms interacting with their environment will have some probability of injury that could lead to veterinary intervention and not all injury is induced by the test article $[13,14]$.

9. Medical treatments are oftentimes based on staff experience. Historical memory and experience by staff veterinarians are an asset-use it. There is almost always more than one effective therapy; the choice of treatments should be discussed prior to study initiation as much as possible based on what is known about the test article. In the immediate moment of intervention it is the SD and staff veterinarian that are present and have the depth of knowledge and clinical experience to make the decision, give them the decision making approval. If the Sponsor disagrees, that animal may be identified for post hoc review of data consistency with group cohorts and the a priori strategic plan to exclude the animal's data with clear transparency to avoid suspected bias. 9a) An outlier is an observation that appears to deviate markedly from other observations in the same group of 
animals on study (for example a food consumption value that is negative). Identification of potential outliers is important for the following reasons. An outlier may indicate compromised data and if it can be determined that an outlying point is in fact erroneous, then that value can be removed from the analysis (or corrected if possible) but this option should be declared within the statistics section of the initial protocol. In some cases, it may not be possible to determine if an outlying point is bad data. Outliers may be due to random variation or may indicate something scientifically relevant. In any event, we typically do not want to simply suppress the outlying observation. However, if the data contains significant outliers, we may need to consider the use of robust statistical techniques. A number of formal outlier tests have proposed in the literature [15].

10. All parties involved in the conduct of animal research have equal roles in conducting quality research which starts with study design. Quality is also the responsibility of the technicians who conduct daily observations, room cleaning, cage changes, as well as a Study Monitor who may reside at a pharmaceutical company four states away from the laboratory. As the adage goes, "quality is job one".

\section{Conclusion}

We write this review from experience as we have been involved at every level of nonclinical safety assessment. We have been active members of an IACUC and served as the SD on hundreds of studies. We acknowledge our own part in some of the strife that has occurred during the paradigm shift in research philosophy. With respect to animal distress and well-being, as an industry we aren't there yet. We encourage everyone to open discussions or dialogue on how to iron out old wrinkles that still exist in the drug development industry as a whole and in CROs, specifically. We are hopeful, we are on our way and the tide has changed but we can do more. We encourage our colleagues to join the conversation.

\section{References}

1. Buchanan JR, Burka LT, Melnick RL (1997) Purpose and Guidelines for Toxicokinetic Studies within the National Toxicology Program. Environ Health Perspect 105: 468-471.

2. Akaberi M, Hosseinzadeh H (2016) Grapes (Vitis vinifera) as a Potential Candidate for the Therapy of the Metabolic Syndrome. Phytother Res 30: 540-556.
3. Ehrenpreis S, Kimura I, Kobayashi T, Kimura M (1987) Histamine release as the basis for morphine action on bile duct and sphincter of Oddi. Life Sci 40: 1695-1698.

4. Hamelin BA, Bouayad A, Méthot J, Jobin J, Desgagnés P, et al. (2000) Significant interaction between the nonprescription antihistamine diphen-hydramine and the CYP2D6 substrate metoprolol in healthy men with high or low CYP2D6 activity. Clin Pharmacol Ther 67: 466-477.

5. Mattson JL (1994) A neurotoxicological definition of adverse. In: Weiss B, O'Donoghue JL. Neurobehavioral Toxicity: analysis and interpretation. Pp: 41-46. Raven Press, New York.

6. MacPhail RC (1994) Behavioral analysis of neurotoxicology. In: Weiss B, O'Donoghue JL. Neurobehavioral Toxicity: analysis and interpretation. Pp: 7-18. Raven Press, New York.

7. Recognition and alleviation of distress in laboratory animals (2008) National Research Council Committee on Recognition and Alleviation of Distress in Laboratory Animals. Washington D.C, The National Acadamies Press.

8. Guidelines for the care and use of mammals in neuroscience and behavioral research (2003) National Research Council (US) Committee on Guidelines for the Use of Animals in Neuroscience and Behavioral Research. Washington D.C, The National Acadamies Press.

9. Animal Welfare Act (AWA) (2017) United States Department of Agriculture (USDA). USC (United States Code), Title 7, Chapter 54, $\$ 2131-2159$.

10. CFR-Code of Federal Regulations Title 21 (2017) United States Food and Drug Administration (FDA). Good Laboratory Practice (GLP) Regulations, Title 21 (Food and Drugs), Chapter 1 (Food and Drug Administration, Department of Health and Human Service), Subchapter A (General), Part 58, Good Laboratory Practice for Nonclinical Laboratory Studies, Code of Federal Regulations.

11. US Code: Title 42 -The Public Health And Welfare (PHSA) (2017) Chapter 6 (Public Health Service), Subchapter I (Administration and Miscellaneous Provisions).

12. 9 CFR Chapter I, Subchapter A - Animal Welfare (2017) United States Department of Agriculture (USDA).

13. Soiefer AI, Rauckman EJ (2001) Toxicity assessment with single chemical exposures. In: Jacobson-Kram D, Keller KA. Toxicology testing handbook: Principles, applications and data interpretation. Pp: 19-32. Marcel Dekker, Inc., New York.

14. Banks C, Keller KA (2001) Multidose toxicity and carcinogenicity studies. In: Jacobson-Kram D, Keller KA. Toxicology testing handbook: Principles, applications and data interpretation. Pp: 33-72. Marcel Dekker, Inc., New York.

15. Winer BJ, Brown DR, Michels KM (1991) Statistical principles in experimental design. (3rd Edn), McGraw Hill, Boston. 